\title{
Indicadores de desempeño académico como predictores de captación de recursos financieros
}

\author{
Indicators of Academic Performance as Predictors of \\ Financial-Resource Raising
}

\author{
Martín P. Pantoja-Aguilar (D) https://orcid.org/0000-0002-8337-5427 \\ Universidad de Guanajuato, México,pantojam@ugto.mx
}

\begin{abstract}
This study was conducted on State Public Universities (SPU) in Mexico to ascertain the possible existence of a correlation between their performance in their indicators of academic excellence and the level of allocation of financial resources obtained from the main sources of funding from the federal government. By means of the determination of the academic and financial indicators used by the main international and national organisms, a methodological proposal of statistical analysis was constructed to evaluate the academic performance of SPU and its correlation with the reception of financial resources. The results showed that there is insufficient evidence to infer that obtaining better academic results in Mexican SPU is a reliable predictor to obtain greater financial resources. This demonstrates the need for a clear policy in the allocation of resources to institutions.
\end{abstract}

Key words: Higher education, funding, quality, indicators, policies.

Recepción:

03/02/2018

Aprobación:

$10 / 10 / 2018$
Resumen: El presente estudio abordó el ámbito de las Universidades Públicas Estatales (UPE) en México, para determinar la posible existencia de una correlación entre el desempeño de estas en los indicadores de excelencia académica y el nivel de asignación de recursos financieros obtenidos de las principales fuentes de financiamiento del gobierno federal. Mediante la determinación de los indicadores académicos y financieros utilizados por los organismos internacionales y nacionales más importantes se construyó una propuesta metodológica de análisis estadístico, con el fin de evaluar el desempeño académico de las UPE y su correlación con la consecución de recursos financieros. Los resultados mostraron que no existe suficiente evidencia para inferir que la obtención de mejores resultados académicos en las UPE mexicanas es un predictor confiable de mejor captación de dichos recursos. De esta manera se expone la necesidad de una política clara en la asignación de recursos a las instituciones.

Palabras clave: educación superior, financiamiento, calidad, indicadores, políticas. 


\section{Introducción}

Cada día se reconoce con mayor interés el importante papel que juega la gestión administrativa y su liderazgo en el desempeño efectivo de las tres funciones sustantivas en las Instituciones de Educación Superior (IES). En el caso de las universidades, Carrasco (2017: 109) señala que dicho papel se basa en que "el concepto de gobernanza universitaria, de este modo hace referencia a los procesos de gestión y administración universitaria y al análisis del papel directivo en toma de decisiones dentro de las organizaciones universitarias". Esto es complementado por Ganga et al. (2015) al afirmar que "se tiene como premisa indiscutida que la gestión del gobierno universitario es uno de los factores determinantes para mejorar el quehacer administrativo y, desde ahí, las tareas académicas".

Sin embargo, Brunner (2011) explica que

el desafío es encontrar un principio de efectividad que facilite implementar cotidianamente las decisiones estratégicas y administrar la organización de manera que se asegure la continuidad de sus funciones, obtener los recursos necesarios para ella y producir resultados satisfactorios para las diversas partes interesadas en medio de un entorno turbulento y a veces hostil (Brunner, 2011: 140).

Al enfocarse en la gestión de recursos para las instituciones, Khem (2011) menciona que una de las principales nociones que integran el concepto de gobernanza es la transparencia. Pero dicha noción debe llevarse a cabo teniendo como eje rector el principio señalado por Berrios et al. (2009: 527) sobre una gestión universitaria que ejerza "una acción autónoma y responsable, donde los actores se acojan al principio de corresponsabilidad y además en los niveles internos y externos de la organización se establezca una gestión para la rendición de cuentas transparente de los procesos".

Esto sin duda será una de las determinantes de éxito en la búsqueda de recursos que realicen las IES, sobre todo las instituciones públicas que dependen, en gran medida y sustancialmente, de los recursos que provee el gobierno federal, y hasta cierto punto el gobierno estatal. El presente estudio se centra en determinar si el nivel de excelencia académica -medida en estándares o indicadores - tiene alguna correlación con el éxito de la gestión administrativa, reflejada en el nivel de captación de recursos financieros para las Universidades Públicas Estatales (UPE) en México. La investigación se fundamenta en la identificación de indicadores de excelencia académica utilizados por los diversos programas nacionales de reconocimiento a la calidad académica y de apoyo financiero a las UPE, los usados por las diferentes organizaciones nacionales cuyo objetivo primordial es reconocer la excelencia en 
la calidad académica de las instituciones y por los que ocupan los principales sistemas de clasificación de universidades de clase mundial, o los denominados rankings (World University Rankings).

Asimismo, se tomó la metodología desarrollada por Pantoja (2016), para determinar el mejor escenario de asignación de peso e impacto en las distintas variables que se utilizaron en el estudio. Este generó como propuesta original una base de indicadores de calidad académica de las UPE en México, que no se encontraba organizada, actualizada y disponible de manera integral en ninguna de las instancias de gobierno u organismos de reconocimiento a la calidad de las instituciones educativas o asociaciones de universidades. $\mathrm{Al}$ análisis de los indicadores de desempeño académico se incorpora la variable de los recursos financieros captados por las UPE en los diferentes fondos disponibles a concurso en el ámbito federal, ${ }^{1}$ con el propósito de llevar a cabo un análisis de correlación que permitiera determinar la validez, o no, de la hipótesis planteada.

El estudio fue aplicado a cada variable académica en relación con cada fuente de financiamiento y con la suma total de las fuentes. La tendencia con dicha metodología fue corroborada en sus resultados: no se encontró correlación significativa entre el nivel de desempeño académico de las UPE y la mejor asignación de recursos financieros federales. La anterior tendencia se mantuvo incluso cuando se incorporaron dos variables alternativas basadas en el contexto económico de las UPE, suponiendo que pudieran ser causa de la asignación de recursos. Con ello se demostró que la conclusión del estudio cuenta con un significativo nivel de confianza sobre la prueba de la hipótesis.

\section{Revisión literaria}

\section{Calidad académica y sus indicadores de desempeño}

El tema de la evaluación de la calidad académica ha sido desde su origen motivo de debate en el ámbito universitario; y la determinación de indicadores que reflejen el nivel de calidad académica de las instituciones es aún más controvertida. En México, las UPE han adoptado políticas federales de calidad académica complementándolas con las reglas de evaluación de los diversos organismos acreditadores nacionales e internacionales, incluyendo

1 Este artículo fue elaborado gracias a la investigación financiada por la Universidad de Guanajuato, a través del programa de Doctorado en Liderazgo y Dirección de Instituciones de Educación Superior de la Universidad Anáhuac. 
las de los rankings (clasificaciones) internacionales y algunos criterios propios de cada UPE. Silva (2013) reafirma lo anterior al mencionar que las

políticas son de dos tipos: las generadas por la propia institución -en este caso la universidad- y las que se derivan del nuevo entorno de políticas públicas de la educación superior, que suponen una suerte de esfuerzos de "adaptación incremental" de las universidades a las exigencias y reglas contenidas en dichas políticas, expresadas empíricamente en los diversos programas federales que involucran recursos extraordinarios, reconocimientos o prestigio para las propias universidades, a los grupos académicos (cuerpos académicos) o a los individuos (profesorado y estudiantes) (Silva, 2013: 87).

Hoy en día existen diversos rankings nacionales e internacionales que se han convertido en la pauta para reconocer la calidad en las IES, e incluso para la asignación de recursos o de apoyos diversos. Martínez-Rizo (2011: 95) señala que las "serias deficiencias metodológicas que tienen los hace sumamente inapropiados como herramientas para evaluar de manera confiable la calidad global de las instituciones a las que se refieren". Sin embargo, en México, como en muchos países del mundo, los rankings y sus deficiencias siguen siendo aplicados como herramientas para detectar la calidad en las IES. Muchos de sus indicadores son utilizados por los sistemas nacionales de evaluación al elaborar sus criterios, y también por los gobiernos al elaborar políticas federales que incluyen el otorgamiento de recursos financieros, con base en el desempeño de dichos indicadores. En la actualidad hay una gran variedad de rankings a lo largo del mundo. Cada uno mide de forma diferente la calidad académica a través de indicadores previamente elegidos por sus creadores. Los resultados en un determinado ranking para una IES pueden ser muy distintos para la misma en otro ranking.

Este fenómeno se produce porque cada ranking tiene objetivos, metodologías y contextos diferentes. Por esta y otras razones,

las mediciones o rankings (en inglés) son motivo de debate en los claustros universitarios debido a las metodologías, que según algunos, no toman en cuenta las diferencias existentes entre las universidades del mundo. No obstante, estas clasificaciones se han convertido en una herramienta adecuada que utilizan las universidades para aumentar la visibilidad y la competitividad (Mattar et al., 2013).

En contraste, nuevamente Martínez-Rizo (2012) menciona que han surgido rankings que en lugar de hacer un ordenamiento global, simplemente realizan un análisis más detallado y no asignan lugares individuales, sino que establecen grupos de desempeño (alto, medio o bajo) y con ello se eliminan algunas de las inconsistencias de los rankings individuales en los cuales las posiciones cambian mucho de un año a otro. 
Los tres principales rankings internacionales más reconocidos en el mundo por los diversos autores son: el Academic Ranking of World Universities (ARWU) o Ranking de Shanghai, elaborado por la Universidad Jia Tong, de China; el World University Ranking, del Times Higher Education Suplement (THE), de Inglaterra; y el Ranking Mundial de Universidades en la Web (Webometrics), del Laboratorio de Cibermetría del Consejo Superior de Investigaciones Científicas de España. En esta clasificación son coincidentes Pusser y Marginson (2013), Martínez-Rizo (2011), Rodríguez (2006), Mattar et al. (2013), Ilera et al. (2013) y Jeremić et al. (2013).

Los tres más citados rankings mundiales utilizan principalmente la información generada por Thomson Reuters, SCImago, los premios Nobel, las medallas Fields, el Majestic SEO, Ahrefs, Google, Google Scholar, Ministerios de Educación, Asociaciones de Universidades, Conferencias de Rectores y la producida por las mismas instituciones evaluadas. La mayoría de las universidades mexicanas no tienen acceso a la clasificación de los tres primeros grandes rankings. Quizá por ello algunos rankings han creado subclasificaciones que obedecen a indicadores y metodologías diferenciadas, y además agrupan IES en su clasificación con un cierto ámbito común o características similares.

Para el caso de Latinoamérica, un ejemplo es el ranking denominado QS World University Rankings, el cual es publicado por la empresa QS de medios, eventos y software en educación. Este ranking tiene un objetivo eminentemente empresarial y no es utilizado con frecuencia como referente por los autores del tema; sin embargo, también ha emitido una subclasificación llamada QS University Rankings: Latin America, la cual ocupa una metodología distinta e incluye a un mayor número de IES mexicanas.

Es indudable que las IES reconocen las deficiencias de los rankings ya mencionados, pero también reconocen que son las reglas del juego actuales, y que si desean acceder a recursos o posicionar su imagen en la arena nacional e internacional deben lograr un buen desempeño en dichos indicadores. Empero, en lugar de incrementar la calidad de las IES, la metodología global actual de la mayoría de los rankings puede causar en la realidad una disminución de la calidad, pues suelen descuidarse áreas académicas importantes que no son valoradas en la metodología de los rankings. En el caso particular de las IES mexicanas, estas no se han organizado y construido un ranking propio, debido al alto costo que significa elaborarlos, lo cual incluye no solamente recursos financieros, sino también horas hombre de personal especializado.

Sin embargo, existen programas federales que mediante su metodología marcan la pauta para que las universidades, lo prefieran o no, entren a la dinámica 
del enfoque de los rankings. Un ejemplo de ello lo confirman Comas et al. (2014), al mencionar que uno de los principales programas federales de financiamiento, el denominado "Programa Integral de Fortalecimiento Institucional" (PIFI), utiliza

un conjunto de indicadores para modelar las características que debe tener una institución de educación superior de calidad, tomando como referencia las llamadas universidades de investigación y, con base en ello, establecer un orden jerárquico (ranking) para obtener recursos económicos adicionales (Comas et al., 2014).

En contraparte, se encuentran los procesos de acreditación y certificación de las IES, conducidos por los organismos acreditadores y los comités de pares. Los indicadores que dichos entes ocupan son seleccionados respecto a la disciplina y al campo profesional de la rama académica que está siendo evaluada. Muchos de sus indicadores pueden ser coincidentes con los establecidos en los programas federales, pero en diversas ocasiones existen indicadores o prioridades que los organismos y los comités solicitan a las IES, y cuyo cumplimiento puede contradecir muchas veces aquellos solicitados en los programas federales. Esta contradicción plantea un gran reto para las IES, ya que el cumplimiento de cada indicador es relevante por su impacto, pero es imposible cumplir con todos, y la IES debe decidir dónde concentrar sus recursos y esfuerzos, a pesar de que ello signifique un posible impacto negativo en alguna evaluación como consecuencia.

Por ello, es necesario armonizar criterios para que haya una mayor equidad en la asignación de recursos, la cual debe ser construida incluso desde el Plan Nacional de Desarrollo (PND) del país. El actual PND (2013-2018) cuenta con cinco metas nacionales o "ejes", entre las que se considera la meta III "México con Educación de Calidad", la cual incluye diversas estrategias y líneas de acción que en el caso de la educación superior concentra un fuerte componente hacia el desarrollo científico y tecnológico, y la innovación. Por eso, las IES se ven orientadas a trabajar en conseguir dichos indicadores y en enfocar sus esfuerzos en la investigación si desean gestionar recursos adicionales que les permitan desarrollarse. Muestra de ello son las políticas y criterios de varios programas federales, incluyendo los del Consejo Nacional de Ciencia y tecnología (Conacyt), que asignan mayor valor o puntaje a las iniciativas que reflejan impacto en el PND 2013-2018, privilegiando los componentes de investigación por encima de otros de la docencia y la vinculación. 


\section{La gestión universitaria y el estilo de liderazgo}

La gobernabilidad y la gobernanza de las universidades han estado siempre enmarcadas en una serie de factores internos y externos que las impactan y definen. Acosta (2014) menciona que "la gobernabilidad institucional significa la capacidad del sistema de gobierno universitario para atender las demandas internas y externas a la organización”. En tanto, Aguilar (citado por Acosta, 2014) señala que "la gobernanza institucional, por su parte, se define como una capacidad organizacional, de gestión y coordinación, en la que una comunidad es capaz de acordar objetivos comunes, coordinar acciones para alcanzarlos y generar las estrategias institucionales más adecuadas para conseguirlos". Según Rodríguez (2014), "un gobierno universitario debería ser capaz de conducir a la institución por el camino del compromiso ético y político con la verdad, la justicia y la solidaridad"; y cuestiona más adelante si dicho gobierno puede lograr tales objetivos mediante una estructura piramidal de autoridad y control.

Este cuestionamiento debe darse dentro de una realidad de participación colegiada en las universidades y en un ambiente de liderazgo conducido por grupos de académicos profesionales especializados, o por un grupo de científicos (Rodríguez, 2014). Sin embargo, cabe resaltar que, como indican Castro y Tomás (2016), el gobierno universitario ha de transitar en un modelo actual, con tres rasgos relevantes que influyen directamente en la gobernabilidad y la gobernanza institucional. El primero es la estructura matricial existente en las líneas de autoridad; el segundo es la no profesionalidad de los académicos que ocupan los cargos de gestión; y el tercero, la transitoriedad del cargo. En todo caso, Sánchez (2013) identifica en su estudio que las buenas prácticas de gestión que se llevan a cabo por el gobierno universitario y su comunidad "son aquellas en las que se detecta un liderazgo distribuido, procesos colegiados de toma de decisiones, así como un equilibrio entre lo pedagógico y lo administrativo".

Por ello, la capacidad de gestión y liderazgo de un rector es un factor importante para la negociación a nivel interno y externo, y este último incluye la negociación con las autoridades educativas federales. Por eso, Badillo et al. (2015) señalan que "el liderazgo surge en las relaciones humanas que se desarrollan dentro y fuera de las IES y representa un nuevo tipo de coordinación e influencia”, ofreciendo una posibilidad de negociación efectiva generada por el estilo de liderazgo transformacional que debiera prevalecer entre los rectores. Esto es lo que explicaron Kouzes y Posner (2003), al definir el liderazgo como el arte de movilizar a otros para que quieran luchar por las aspiraciones compartidas, en este caso las de una comunidad universitaria. 
Sin embargo, podemos observar que en las IES mexicanas prevalece el estilo de liderazgo transaccional, y algunas veces autocrático, con un enfoque centralizado en la toma de decisiones, predominando el liderazgo basado en las características de su líder. Por eso, Sánchez et al. (2015) señalan que

dadas las múltiples críticas recibidas por esta clásica visión del liderazgo, en los últimos años ha ido consolidándose otra línea de pensamiento sobre el tema que prioriza la atención al contexto y a los estilos de liderazgo, entendido éste como práctica, por encima de una consideración individualista y descontextualizada de las características personales del líder (Sánchez et al., 2015).

Ante la nueva tendencia, es relevante destacar que la combinación del contexto y las características del líder requieren de un correcto nivel de práctica ética por parte de los rectores. Así lo demuestran Walumba et al. (2008) en su estudio, al identificar que sí existe una correlación positiva entre un liderazgo auténtico y la ética en el liderazgo, indicando el liderazgo auténtico con líderes con conciencia de sí mismos, transparencia en las relaciones, una conducta auténtica y un procesamiento equilibrado de la información. Dichas características son reafirmadas por Bass y Steidlmeier (1999), al explicar que un agente moral es evaluado como digno de alabanza o culpable a la luz de tres consideraciones primarias: su nivel de conciencia de desarrollo; grado de libertad efectiva y probidad de intención. Con base en Bass y Steidlmeier (1999), se puede concluir que el liderazgo de los rectores debe apegarse a un marco ético sustentado en la autenticidad, la transparencia y la conducta honesta. Un liderazgo compartido que permita la toma de decisiones colegiada y garantice la gobernabilidad y la gobernanza de la institución; toda vez que Abad (1997) apunta que "aquellos métodos que permitan igual participación en el proceso de toma de decisiones pueden lograr mucho mayor aceptación sin poner en peligro la calidad de la decisión".

El enfoque al usuario, el mercado y la sociedad (UMS)

En la historia de la administración y su gestión se identifican tres tipos de enfoque u orientaciones principales: a la tarea, al proceso y al usuario-mercado-sociedad (UMS). En el inicio, de acuerdo con López et al. (2006), "la administración científica fundada por Taylor y sus seguidores constituye el primer intento de formular una teoría de la administración, la preocupación por crear una ciencia de la administración y el énfasis en las tareas". Posteriormente, las teorías modernas de la administración evolucionan y surge un nuevo enfoque a procesos, definido por Mallar (2010) como "un nuevo 
concepto de estructura organizativa que considera que toda organización se puede concebir como una red de procesos interrelacionados o interconectados, a la cual se puede aplicar un modelo de gestión denominado Gestión basada en los Procesos (GBP)". Finalmente, como producto de la administración sistémica propuesta por Ackoff (2008), y de acuerdo con el organigrama UMS de aplicación a las organizaciones desarrollado por Nosnik (2013), surge este último enfoque que responde con mayor calidad a las necesidades de las funciones competitivas (comercial y producción) de una empresa u organización, mediante el apoyo de las funciones efectivas (administración y finanzas, recursos humanos y sistemas). En la Figura $1^{2}$ se puede observar que la orientación UMS utiliza la delegación para la administración de recursos, aplicando la cultura de servicio interno entre áreas y equipos: cliente-proveedor. Dicha cultura se aplica a las variables efectivas en apoyo a las competitivas, buscando satisfacer al mercado que forma parte de la sociedad.

El enfoque UMS usado en el ámbito de las instituciones, como las universidades, posibilita analizar dentro del concepto de cadena de valor la relación deseable entre la actividad administrativa (funciones efectivas) y la académica (funciones competitivas), siendo la primera el respaldo de la segunda. En este caso, el modelo UMS se puede ocupar para clasificar los indicadores de excelencia académica de las universidades, respondiendo con calidad a las necesidades de llevar a cabo las funciones competitivas de la universidad: docencia, investigación y extensión (difusión de la cultura) en la sociedad.

\section{Los programas federales de financiamiento a las UPE}

El motor primordial utilizado por el gobierno federal para incentivar los cambios en las UPE han sido los programas federales de financiamiento para la operatividad y desarrollo, que establecen en sus metas y objetivos los indicadores de excelencia académica considerados primordiales. Estos indicadores han sido, incluso, variables en el tiempo y muchas de las veces obedecen a convicciones personales de los directivos de la educación superior federal. Los principales programas federales de financiamiento a que actualmente tienen acceso son:

2 Las figuras y las tablas se encuentran en el Anexo, al final del presente artículo (Nota del editor). 
1. Convenio de cofinanciamiento del subsidio ordinario (Ordinario).

2. Programa de Fortalecimiento de la Calidad en Instituciones Educativas (Profocies), vigente hasta el año 2016.

3. Fondo para el Reconocimiento de Plantilla en las Universidades Públicas Estatales (Plantilla), vigente hasta el año 2015.

4. Fondo de Apoyo para Reformas Estructurales de las UPE (Reformas).

5. Fondo para Elevar la Calidad de la Educación Superior (FECES).

6. Programa de Estímulos al Desempeño del Personal Docente (Estímulos).

7. Programa de Expansión de la Oferta Educativa en Educación Media Superior y Superior (Expansión).

8. Fondo de Aportaciones Múltiples (FAM).

9. Programa de Apoyo al Desarrollo de la Educación Superior (PADES).

\section{Metodología}

Para el presente estudio se planteó la siguiente hipótesis:

La obtención de mejores resultados académicos en las UPE mexicanas es un predictor confiable del volumen de recursos financieros ordinarios $y$ extraordinarios que estas universidades captan al ser estudiadas con un enfoque al usuario, al mercado y a la sociedad (UMS).

Cabe mencionar que existe la posibilidad de una relación inversa; esto es, que a medida que las UPE reciben un mayor nivel de ingresos como financiamiento, los resultados en sus indicadores académicos podrían ser mejores. Para esta investigación se decidió optar por la primera reflexión planteada en la hipótesis, ya que está basada en la metodología propuesta por Pantoja (2016), en la tesis doctoral donde realizó pruebas estadísticas a cinco metodologías de análisis, para definir su variabilidad y determinar con ello la combinación que proporciona la menor variabilidad en los resultados, incorporando pesos específicos y normalizadores para el caso de los indicadores académicos. El periodo seleccionado para recolectar información fue de siete años (del 2001 al 2017) y la muestra del estudio incluyó un total de 34 instituciones. Se decidió examinar al grupo de las UPE mexicanas, pues se considera que cuentan con una estructura similar que permite la comparación entre ellas. Dentro de una concepción genérica, los últimos siete años muestran cierta estabilidad, con pocas variaciones entre el número de programas federales disponibles para financiamiento de las UPE y el tipo de indicadores de desempeño solicitados en dichos programas. 
Para el presente estudio, en la Figura 2, Pantoja (2016) adaptó el diagrama original de Nosnik (2013) llevándolo al ámbito de las universidades; dado que no se midieron estructuras de las UPE, esta investigación utilizó el modelo UMS para organizar causalmente indicadores de desempeño académico y de gestión financiera (en bases de datos). En su elemento usuario, se identificó a los alumnos; en el elemento mercado se ubican los productos de las instituciones universitarias (docencia, investigación y extensión o difusión de la cultura), pues el mercado es el receptor de los egresados, los productos de la investigación y los proyectos de extensión universitaria. Finalmente, en el elemento sociedad se coloca a las universidades públicas que toman en cuenta las demandas especiales y de equidad de los miembros de la sociedad, mediante aspectos como inclusión, movilidad y generación de oportunidades.

En la Figura 3 se puede observar que Pantoja (2016), partiendo del enfoque UMS, definió como variable independiente la constituida por el conjunto de indicadores de desempeño académico y competitividad que abarcan las funciones de docencia, investigación y extensión (difusión de la cultura). Por otro lado, la variable dependiente fue establecida por la gestión institucional y su efectividad, reflejada en la captación de recursos financieros. Para la variable independiente se seleccionó un total de 44 indicadores académicos, con base en su disponibilidad y en la confiabilidad de la fuente de información. Los indicadores fueron organizados de acuerdo con el ámbito de influencia: de alumnos, de profesores, de los programas educativos (PE), de la investigación y de los rankings mundiales de universidades (RMU).

En cuanto a los indicadores de desempeño en la gestión administrativa, se sacaron los promedios de los recursos financieros obtenidos en las diversas fuentes de presupuesto ordinario y extraordinario seleccionadas, todo ello dentro del periodo evaluado. Los resultados se concentraron en un listado según el orden provisto por la Secretaría de Educación Pública (SEP) para las UPE. Al final se analizó la correlación entre los resultados de puntaje asignados a los indicadores de desempeño académico y los promedios de recursos financieros obtenidos en los indicadores de gestión administrativa. 


\section{Resultados del estudio}

\section{Análisis de correlación}

Debido a que existen nueve fuentes federales de financiamiento para las UPE, se realizó un análisis correlacional entre la variable independiente puntaje y cada una de las fuentes de financiamiento federales (variables dependientes) recibidas por cada UPE, utilizando el coeficiente de correlación de Pearson $(r)$ :

$$
\rho=\frac{\operatorname{Cov}(x, y)}{\sigma_{x} \sigma_{y}}
$$

Para probar la hipótesis de forma todavía más precisa, adicionalmente se consideró pertinente calcular, a manera de verificación, el coeficiente de correlación de Spearman (rs). Según Hauke y Kossowski (2011), dicho coeficiente evalúa la relación monótona, es decir, no lineal entre dos variables continuas u ordinales. El coeficiente por rangos fue desarrollado por el psicólogo británico Charles Edward Spearman, y de acuerdo con Johnson y Kuby (2012), la fórmula de cálculo es la siguiente:

$$
\rho=1-\frac{6 \sum D^{2}}{n\left(n^{2}-1\right)}
$$

Al igual que el coeficiente de Pearson, el de Spearman se mide por valores que oscilan entre - 1.0 y 1.0, y la diferencia consiste en que la medición de este último confirma si existe correlación en una distribución monótona, esto es, no lineal. Entre más cercano se encuentre el valor de rs a la unidad, mayor será el nivel de correlación, ya sea positiva o negativa (Johnson y Kuby, 2012).

Es importante mencionar que en el presente estudio se buscó evidenciar si el comportamiento de una variable que se definió como independiente (excelencia académica) está positivamente correlacionado, o no, con el comportamiento de una variable definida como dependiente (recursos financieros). Esto no significa encontrar la causalidad de la variable dependiente mediante el coeficiente de correlación, ya que la primera puede ser originada por una serie de factores que serían materia de estudios posteriores y más complejos. Como lo afirma Pearl (2000), es relevante hacer una clara distinción entre parámetros estadísticos y parámetros causales. En los primeros se encuentra la correlación, y entre los segundos se hallan conceptos como la aleatorización y la confusión. 


\section{Prueba de la hipótesis}

En la Tabla 1 se presentan los resultados del análisis de correlación entre la variable independiente puntaje y los distintos programas de apoyo financiero para las UPE. El coeficiente de Pearson $r$ de la variable captación total con un valor de 0.259 hace referencia a la existencia de una relación muy débil que no la explica ni medianamente, declarada por Triola (2013) como moderada en un punto de 0.43 , y que se encuentra un poco más lejano al punto de 0.50, mencionado por Witte y Witte (2015), Cohen (1988), Devore (2012) y Lind et al. (2012). Reforzando lo señalado por Witte y Witte (2015), la lejanía de todos los coeficientes calculados respecto al valor de +1.0 o -1.0 significa que no hay una relación mayor entre las variables, que explique una relación lineal entre ellas.

Asimismo, aclaran que los coeficientes de correlación menores de 0.50 indican que no hay una fuerte relación que explique su correspondencia. Respecto al coeficiente de Pearson $r$ en las variables de los distintos fondos, se puede observar que los resultados arrojaron coeficientes de correlación muy bajos o débiles, abarcando desde un resultado menor de $r=0.071$ en la variable $F A M$, y hasta uno máximo de $r=0.459$ en la variable estimulos, con resultados intermedios de las otras variables, pero tendiendo hacia la baja.

En cuanto al coeficiente de Spearman $r$ s, se nota que no hay una variación significativa entre los resultados comparados con el coeficiente de Pearson $r$. En la mayoría de las ocasiones, el coeficiente de Spearman sube ligeramente en comparación con el de Pearson, y en otros casos, incluso, desciende un poco. Lo anterior confirma que tanto bajo un modelo lineal como bajo un modelo monótono los datos analizados no muestran una correlación fuerte que haga presumir la posible existencia de causalidad en ellos. Al comparar de manera individual el desempeño de las UPE en sus principales indicadores de excelencia académica y agrupados mediante el enfoque UMS contra la captación de recursos en cada fuente federal y estatal, se evidenció una correlación que va de excesivamente baja y hasta correlaciones que pudieran considerarse moderadas, pero que no explican ampliamente una fuerte relación lineal entre variables.

Cabe mencionar que solo en la variable estimulos se obtiene un coeficiente de Pearson de $r=0.459$ y un coeficiente de Spearman de $r s=0.528$, ubicando esta variable en una correlación moderada que permite inferir una mayor correspondencia entre el resultado académico de las UPE y la asignación de recursos financieros, aunque no presenta un nivel de correlación que posibilite afirmar una tendencia positiva para la prueba de la hipótesis. A 
esto se aúna a que el fondo estímulos representa una minoría en el total de la asignación de recursos federales a las UPE, al considerar todos los fondos federales en la variable captación total. En la Tabla 1 se puede ver que el coeficiente de Pearson es de $r=0.259$ y el de Spearman, de $r s=0.205$, lo cual significa una correlación muy débil.

Derivado de lo anterior, se puede inferir que no hay evidencia suficiente para afirmar que el mejor logro de indicadores de desempeño académico en una UPE, mediante un enfoque UMS, es predictor de un mayor nivel de captación de recursos financieros en los programas de presupuesto ordinario y extraordinario. La posibilidad de encontrar causalidad en la variable dependiente generada por la variable independiente sigue siendo muy baja, y no se puede asegurar que una variable genera el comportamiento de la otra, ya que la casualidad entre ellas debe ir acompañada de una correlación alta, sin olvidar que tener esta no necesariamente significa que haya causalidad entre ellas; pero en una correlación moderada o baja se puede presumir que hay muchas menos posibilidades de hallar causalidad directa entre las variables.

Dado que una correlación débil no es un término absoluto, sino un concepto vago, se consideró conveniente tomar en cuenta contextos económicos que pudieran influir en la relación causal directa entre variables. Para ello fueron seleccionadas variables alternativas con la sospecha de su influencia como factores económicos regionales sobre la variable independiente. La sospecha era que estas otras variables alternativas podrían estar igualmente correlacionadas, como lo están las variables dependientes del estudio. Por eso, a manera de prueba, se decidió medir la correlación de la variable independiente (indicadores académicos) con dos variables dependientes elegidas de forma alternativa: la población total por estado (población) y el Producto Interno Bruto por Estado (PIBE).

Dicha información fue correlacionada ubicando a cada UPE en el estado donde proporciona sus servicios académicos (los resultados se muestran en la Tabla 2). Se puede observar que se obtuvieron resultados de $r=0.304$ en la variable población y de $r=0.379$ en la variable $P I B E$, mostrando una correlación lineal moderada con posibilidades muy limitadas de explicar en forma aislada y directa la causalidad de las variables. Pero es interesante resaltar que las variables alternativas, en muchos de los casos, tuvieron un factor de correlación $r$ mayor al de las diversas fuentes de financiamiento, evidenciando que variables aleatorias explican mejor la posible existencia de una correspondencia con los indicadores académicos. Por otro lado, los coeficientes de correlación de Spearman arrojaron en ambas variables alternas un valor menor al coeficiente de Pearson, indicando que bajo una 
medición de correlación que se sospecha monótona las variables alternas muestran una más débil relación.

En este punto surge la posibilidad de una hipótesis alternativa, que explique las decisiones de financiamiento del gobierno federal hacia las UPE, quizá de una manera remedial: esto es, que el gobierno busca remediar el bajo desempeño de algunas UPE mediante la asignación superior de recursos para impulsar sus logros académicos. Por ello, en la Tabla 3 se exponen los resultados del análisis complementario de correlación, reuniendo en tercios a las UPE con base en los resultados de los indicadores de desempeño académico. Dichos resultados fueron agrupados en dos niveles: el tercio de las UPE que obtuvieron el mejor resultado en indicadores académicos y el tercio de las UPE que tuvieron el menor resultado en esos indicadores.

En el tercio de las UPE que consiguieron el puntaje más alto, se notaron, en su mayoría, correlaciones débiles e incluso negativas: a medida que las IES consiguen mejores resultados académicos, reciben una menor cantidad proporcional de recursos financieros federales. Incluso, al calcular el coeficiente de correlación de Spearman, los resultados mostraron muy poca diferencia con el coeficiente de Pearson, ubicándose en un nivel menos que moderado de correlación. Por otra parte, al analizar el tercio de las UPE con los resultados más bajos, se pudo constatar que los coeficientes de Pearson tuvieron en su mayoría valores negativos, y unos pocos positivos, pero de muy bajo nivel; es decir, no se encontró suficiente evidencia de una posible correlación y mucho menos de una causalidad.

$\mathrm{Al}$ aplicar el coeficiente de Spearman, los resultados indicaron varios valores negativos, y en algunos fondos valores positivos, e incluso cercanos a 0.50 , y de 0.60 en el caso de uno de ellos. Aunque algunos de los fondos pudieran inferir una relación moderada, no se puede concluir que haya una correspondencia lineal entre la asignación de recursos y los resultados académicos en este grupo. Más aún, cuando se analizó la variable captación total, se encontró que el coeficiente de Spearman es de valor negativo. En todo caso, es importante que la distribución de recursos responda constantemente a una política federal de asignación de recursos y no solo de forma moderada a unos pocos.

Con base en lo anterior, se puede afirmar que es muy probable que las políticas federales y su asignación de recursos financieros, a través de los programas, estén incentivando exclusivamente a las UPE que han mantenido un bajo desempeño en sus indicadores académicos, y que estén desincentivando a las UPE que han sostenido un desarrollo en sus indicadores de desempeño académico, al asignarles menores recursos, a pesar de mejorar 
su nivel de indicadores. El resultado se convierte en una curva asintótica en la cual se premia con un mayor nivel proporcional de asignación de recursos financieros a las UPE que no han realizado su trabajo académico de manera efectiva y eficiente, medido a través de lo que los propios programas federales han establecido como "los indicadores" de excelencia en este ámbito.

Posteriormente, en dicha curva se encuentra su parte de menor crecimiento, pues las UPE que han efectuado con excelencia su tarea en esta área reciben menor asignación proporcional de recursos financieros. Este último efecto quizá sea generado por algunas otras variables causales, pero sería difícil incluir su análisis dentro del alcance del presente estudio.

Por todo lo antes señalado, la hipótesis originalmente planteada se rechaza, pues no existe suficiente evidencia estadística que permita inferir que la obtención de mejores resultados académicos en las UPE mexicanas sea un predictor confiable del volumen de recursos financieros ordinarios y extraordinarios que estas universidades captan, al ser estudiadas con un enfoque UMS. Se detecta como hipótesis alternativa que existen otros factores económicos que explican la variación en la asignación de recursos financieros a las UPE, y entre ellos se plantea la posibilidad de que el gobierno federal aplique una lógica remedial para las UPE de menor desempeño académico.

\section{Discusión y conclusiones}

El debate que se propone es continuar investigando si, en efecto, los indicadores actualmente vigentes son los que deberían ser exigidos en los programas federales para asignar recursos financieros, o si dichos indicadores deben ser replanteados, de acuerdo con la reflexión nacional de las instituciones educativas, generando consenso sobre los indicadores y la forma como los recursos requieren ser asignados. Sobre todo, al reconocer que muchas veces las asignaciones de recursos se realizan desde la perspectiva y decisión de los funcionarios en turno a cargo de los programas federales, pues muy frecuentemente dichos programas cambian sus requerimientos en cada ejercicio, enfocándose a aspectos particulares que no atienden una planeación integral de desarrollo de las UPE mexicanas.

Otro aspecto importante de investigar a futuro sería la coordinación posible entre los indicadores que de manera particular requieren los organismos acreditadores y certificadores a las instituciones educativas, y aquellos que requieren los programas federales de asignación de recursos. Aunque en principio se pudiera entender que el logro de los primeros indicadores necesita del logro de los segundos, muchas veces se pueden 
encontrar conflictos internos en las UPE mexicanas, para decidir si enfocan sus esfuerzos a conseguir las exigencias de un organismo u otro, o los requerimientos de alguna dependencia federal.

La hipótesis original en el estudio supuso que a medida que las UPE mexicanas obtienen mejores resultados en sus indicadores de desempeño académico, estas captan un mayor nivel de recursos financieros ordinarios y extraordinarios a través de los programas federales. Los resultados del análisis de correlación entre la variable puntaje de las UPE y su captación de recursos financieros en los distintos programas de apoyo federal arrojaron coeficientes de correlación de muy bajos a moderados. Tanto el coeficiente de correlación de Pearson, que mide la existencia de una relación lineal, como el coeficiente de Spearman, que verifica la existencia de una correlación de tipo monótona, o no lineal, confirmaron los resultados y no mostraron grandes variaciones entre ellos.

Del total de diez coeficientes de correlación calculados, siete de ellos fueron identificados como relaciones débiles con valores muy cercanos al cero o menores a 0.30 , mostrando que no hay una relación lineal o monótona significativa que explique ampliamente su existencia, y que por lo tanto no cabe la posibilidad de una causalidad directa entre variables. También se confirmó que no hay evidencia científica suficiente para afirmar que, en México, a medida que las UPE van mejorando en sus indicadores académicos, como retribución, reciben más recursos financieros. Más aún, por el tipo de análisis correlacional conducido, se puede asegurar que tampoco hay evidencia científica suficiente para concluir que un mayor nivel de recursos financieros en una UPE corresponde a un mayor logro en los indicadores académicos.

Esto es muy preocupante para el contexto de la educación superior en México, ya que las evidencias hacen suponer posteriores hipótesis por investigar en el futuro, que serían inquietantes en caso de ser afirmativas. Una de ellas podría ser el suponer que hay factores discrecionales en la asignación de recursos financieros federales, los cuales generan mejoras poco significativas en el desempeño académico de las UPE mexicanas; también sería muy preocupante que se evidenciaran retrocesos.

El estudio de dos variables alternativas seleccionadas de manera azarosa demostró que hay otras razones que no están directamente relacionadas con el financiamiento federal a las UPE que comprueben un nivel similar de correlación lineal o monótona. Esto desacredita todavía más la posibilidad de correspondencia entre lo que el gobierno federal expresa en sus políticas de asignación de recursos financieros y el logro académico de las UPE. Ello queda evidenciado al mostrar que variables como el número de habitantes de 
un estado, o el PIB del mismo, explican, en igual nivel que las variables dependientes del estudio, la posibilidad de correlación, y en un momento dado la posible causalidad entre las variables analizadas. Incluso, al examinar a las UPE mexicanas de forma sectorizada, los resultados descubrieron un nuevo hallazgo en el comportamiento correlacional de las variables estudiadas. Se evidenció que, el subgrupo de las UPE con mejor resultado en los indicadores de desempeño académico tienen factores de correlación muy débiles e incluso la mayoría muestra un resultado inverso (negativo), respecto de la asignación de recursos financieros.

Este hallazgo es preocupante, pues genera evidencia de que, a mayor esfuerzo y logro académico de las UPE, hay una menor asignación proporcional de recursos financieros federales. En el caso del subgrupo de las UPE con los menores resultados en los indicadores de desempeño académico, al inverso del grupo anterior, se produjo evidencia que soporta la existencia de una baja a moderada, y tendiente a significativa, relación tanto lineal como monótona. Esto es, se encontró que a mayor nivel de asignación de recursos financieros hay un menor resultado en los indicadores de desempeño académico, o viceversa. Es inquietante que algunas UPE no consigan el desempeño necesario en sus indicadores de excelencia académica, que refleje el nivel suficiente para brindar educación equitativa como la sociedad lo demanda; a pesar de ello, el presente estudio demostró que muy probablemente reciben una asignación de recursos proporcionalmente mayor a aquellas que están realizando la tarea académica con un nivel de excelencia. No obstante, se reconoce la importancia que tienen las UPE para el Estado mexicano, sobre todo para las regiones donde están ubicadas, pero es imperativo tomar acciones que posibiliten que sus egresados puedan competir con equidad en el mundo laboral y avancen profesionalmente.

En resumen, la evidencia científica reunida en esta investigación genera la preocupante reflexión sobre el hecho de que a mayor esfuerzo y logro en el desempeño académico de las UPE mexicanas, estas son menos reconocidas con una menor asignación proporcional de recursos financieros. Por otro lado, a pesar de un desempeño académico mediocre, un grupo de las UPE está siendo mejor reconocido con la asignación proporcional de dichos recursos. $\mathrm{O}$ viceversa, a pesar de que un grupo de UPE recibe una menor cantidad proporcional de recursos económicos federales, están consiguiendo mantener y desarrollar indicadores de desempeño académico de excelencia. Sin embargo, a medida que a un grupo de UPE se les inyecta un mayor nivel de recursos económicos, estas siguen manteniendo un desempeño mediocre y sin mejora en los resultados académicos. En cualquiera de las interpretaciones 
anteriores, la conclusión es alarmante para la educación superior pública en el país, y por ello se detecta la gran necesidad de continuar investigando el tema, identificando otras variables que impactan dicho comportamiento.

Tal hallazgo conlleva a otras hipótesis alternativas. Una de ellas establece que quizá la asignación de financiamiento por parte del gobierno federal sigue una lógica remedial en la cual este, buscando mejorar el desempeño académico de dichas instituciones, les otorga mayores recursos para que se fortalezcan. Asimismo, se incluye el supuesto de que las UPE con alto desempeño académico pueden tener acceso a otras fuentes de financiamiento nacionales e internacionales. En todo caso, es importante investigar si la posible lógica remedial ha surtido efectos positivos o estancamiento y retroceso; o en las UPE de alto desempeño, cuáles han sido los factores de impacto que han favorecido alcanzar sus indicadores de desempeño académico, a pesar de la menor asignación proporcional de recursos. Dichas hipótesis alternativas serían dignas de un estudio posterior, donde la variable tiempo podría responder estas incógnitas.

Los resultados de esta investigación muestran que las políticas y lineamientos utilizados por el Gobierno de la República para asignar recursos financieros a las UPE mexicanas no corresponden de manera correlacional ni causal, y en todo caso justa, al esfuerzo de la mejora académica que las instituciones emprenden y que se evidencia en un mejor resultado de indicadores de desempeño académico. Esta situación es por demás controversial, pues los líderes de las UPE mexicanas deben gestionar ante el gobierno federal el establecimiento de políticas y lineamientos claros y equitativos, que premien dicho esfuerzo.

La asignación de recursos financieros debería responder a estos logros de forma transparente y justa. Es muy importante que el Gobierno de la República, las asociaciones de universidades y los organismos certificadores y acreditadores de México reconozcan la relevancia de contar con una política nacional de calidad académica para la educación superior, que armonice las distintas fuentes de financiamiento para las UPE mexicanas e incentive a estas a conseguir los principales indicadores de excelencia académica.

\section{Referencias}

Abad, Isabel Ma. (1997), "El líder y la toma de decisiones: Análisis a través del modelo normativo y su revisión”, en Cuadernos de Ciencias Económicas y Empresariales, vol. 21, núm. 33, España: Dialnet.

Ackoff, Rusell (2008), El Paradigma de Ackoff: Una administración sistémica, México: Limusa Wiley. 
Acosta, Adrián (2014), "Gobierno universitario y comportamiento institucional: la experiencia mexicana, 1990-2012”, en Bordón. Revista de pedagogía, vol. 66, núm. 1, España: Sociedad Española de Pedagogía.

Badillo, Rosalba et al. (2015), "Liderazgo de los rectores frente a la 'tercera misión' de la Universidad. Visiones globales, miradas locales”, en Revista Mexicana de Investigación Educativa, vol. 20, núm. 65, México: Consejo Mexicano de Investigación Educativa.

Bass, Bernard y Steidlmeier, Paul (1999), "Ethics, character, and authentic transformational leadership behavior", en The leadership quarterly, vol. 10, núm. 2, Estados Unidos: Science Direct.

Brunner, José (2011), “Gobernanza Universitaria: Tipología, dinámicas y tendencias”, en Revista de Educación, núm. 355, mayo-agosto, España: Ministerio de Educación y Formación Profesional español.

Berrios, Olecy et al. (2009), "Modelo gerencial en el marco de la transformación universitaria", en Revista de Ciencias Sociales, vol. 15, núm. 3, Venezuela: Universidad del Zulia.

Carrasco, Alexandra (2017), "Los nuevos modelos de gobernanza universitaria. El caso de la unión europea como organismo supranacional que configura el sistema universitario español", en Journal of Supranational Policies of Education, España: Universidad Autónoma de Madrid.

Castro, Diego y Tomás, Marina (2016), "El gobierno y la gestión de la universidad: estudio de los órganos unipersonales”, en Estudios sobre Educación, vol. 19, España: Universidad de Navarra.

Cohen, Jacob (1988), Statistical power for the behavioral sciences, Estados Unidos: Earlbaum.

Comas O. et al. (2014), "El PIFI en las universidades públicas: de la decisión racional a la legitimidad institucional", en Revista de la Educación Superior, vol. 43, núm. 69, México: Asociación Nacional de Universidades e Instituciones de Educación Superior.

Devore, Jay (2012), Probabilidad y estadistica para ingenierias y ciencias, México: Cengage Learning.

Ganga, Francisco et al. (2015), "Teoría de agencia (TA): supuestos teóricos aplicables a la gestión universitaria”, en Innovar, vol. 25, núm. 57, Colombia: Universidad Nacional de Colombia.

Hauke, Jan y Kossowski, Tomasz (2011), “Comparison of values of Pearson's and Spearman's correlation coefficients on the same sets of data", en Quaestiones geographicae, vol. 30, núm. 2, Polonia: Adam Mickiewicz University.

Ilera, José et al. (2013), "Evaluación de la accesibilidad de páginas web de universidades españolas y extranjeras incluidas en rankings universitarios internacionales", en Revista Española de Documentación Cientifica, España: Instituto de Estudios Documentales sobre Ciencia y Tecnología. DOI: 10.3989/redc.2013.1.913.

Jeremić, Veljco et al. (2013), "Proportion of Collaborative Publications as an Ultimate Indicator of Leiden 2013 World Best Universities Rankings", en Management (18200222), Serbia: Universidad de Belgrado. DOI: 10.7595/management.fon.2013.0020.

Johnson, Robert y Kuby, Patricia (2012), Estadistica Elemental, México: Cengage Learning. Khem, Barbara (2011), La gobernanza en la educación superior: sus significados y su relevancia en una época de cambios, España: Ediciones Octaedro.

Kouzes, James y Posner, Barry (2003), The Leadership Challenge, Estados Unidos: Josey-Bass- Wiley.

Lind, Douglas et al. (2012), Estadistica aplicada a los negocios y la economía, México: McGraw Hill.

López, María et al. (2006), "Las organizaciones y la evolución administrativa", en Scientia et Technica, México: Universidad Tecnológica de Pereira. 
Mallar, Miguel (2010), “La Gestión por Procesos: un enfoque de gestión eficiente”, en Visión de futuro, vol. 13, núm. 1. Disponible en: http://www.scielo.org.ar/scielo. php?script=sci_arttext\&pid=S1668-87082010000100004\&lng=es\&tlng=es. $[23 \mathrm{de}$ mayo de 2018].

Martínez-Rizo, Felipe (2012), "Impactos deseables e indeseables en las Universidades de América Latina”, en Presencia e impacto de los rankings en las universidades de América Latina, México: Universidad Nacional Autónoma de México.

Martínez-Rizo, Felipe (2011), "Los rankings de universidades: una visión crítica”, en Revista de la Educación Superior. Disponible en: http://www.redalyc.org/articulo. oa? id=60420223004 [12 de febrero de 2016].

Mattar, Salim et al. (2013), "Análisis de las universidades colombianas de acuerdo con el ranking SCImago 2010-2012", en Revista MVZ (Medicina Veterinaria y Zootecnia). Disponible en: http://go.galegroup.com/ps/i.do?id=GALE\%7CA346007460\&v $=2.1 \& u=$ ugto $01 \& i t=r \& p=G P S \& s w=w \& a s i d=934895 a 8 f 04 b d 09933605823 \mathrm{~d} 2$ 5 f2444 [23 de febrero de 2016].

Nosnik, Abraham (2013), Modelo del Hexágono de Responsabilidades, manuscrito inédito.

Pantoja, Martín (2016), Indicadores de desempeño académico como predictores de captación de recursos financieros en las universidades públicas estatales (UPE), México: Universidad Anáhuac.

Pearl, Judea (2000), Causality. Models, Reasoning and Interference, Estados Unidos: Cambridge University Press.

Plan Nacional de Desarrollo (PND) (2013-2018), Plan Nacional de Desarrollo 20132018, México: Gobierno de la República. Disponible en: http://pnd.gob.mx/ [13 de octubre de 2015].

Pusser, Brian y Marginson, Simon (2013), “University Rankings in Critical Perspective”, en Journal of Higher Education, vol. 84, núm. 4, Reino Unido: Taylor and Francis.

Rodríguez, Roberto (2006), "Rankings universitarios ¿Un oscuro objeto de deseo? Tercera y cuarta parte”, en Suplemento Campus del Diario Milenio. Disponible en: http:// robertorodriguezgomez.blogspot.mx/p/indice-cronologico.html $[18$ de enero de 2016].

Rodríguez, Sebastián (2014), "El gobierno de las universidades: de la reflexión a la acción”, en Bordón, Revista de pedagogía, vol. 66, núm. 1, España: Sociedad Española de Pedagogía.

Sánchez, Marita (2013), "Buenas prácticas de gobierno y gestión en la universidad”, en Teoría de la Educación, Revista Interuniversitaria, vol. 25, núm. 1, España: Universidad de Salamanca.

Sánchez, Marita et al. (2015), "Liderazgo en la universidad: perfil de mujeres y hombres gestores académicos", en Revista Iberoamericana sobre Calidad, Eficacia y Cambio en Educación (REICE), vol. 12, núm. 5, España: Universidad Autónoma de Madrid

Silva, Adrián (2013), "Políticas, actores y decisiones en las universidades Públicas en México: un enfoque institucional", en Revista de la Educación Superior, vol. 42, núm. 165, México: Asociación Nacional de Universidades e Instituciones de Educación Superior. Triola, Mario (2013), Estadistica, México: Pearson.

Walumba Fred et al. (2008), "Authentic Leadership: Development and Validation of a Theory-Based Measure", en Journal of Management, vol. 34, núm. 1, Estados Unidos: University of Nebraska.

Witte, Robert y Witte, John (2015), Statistics, Estados Unidos: Wiley. 


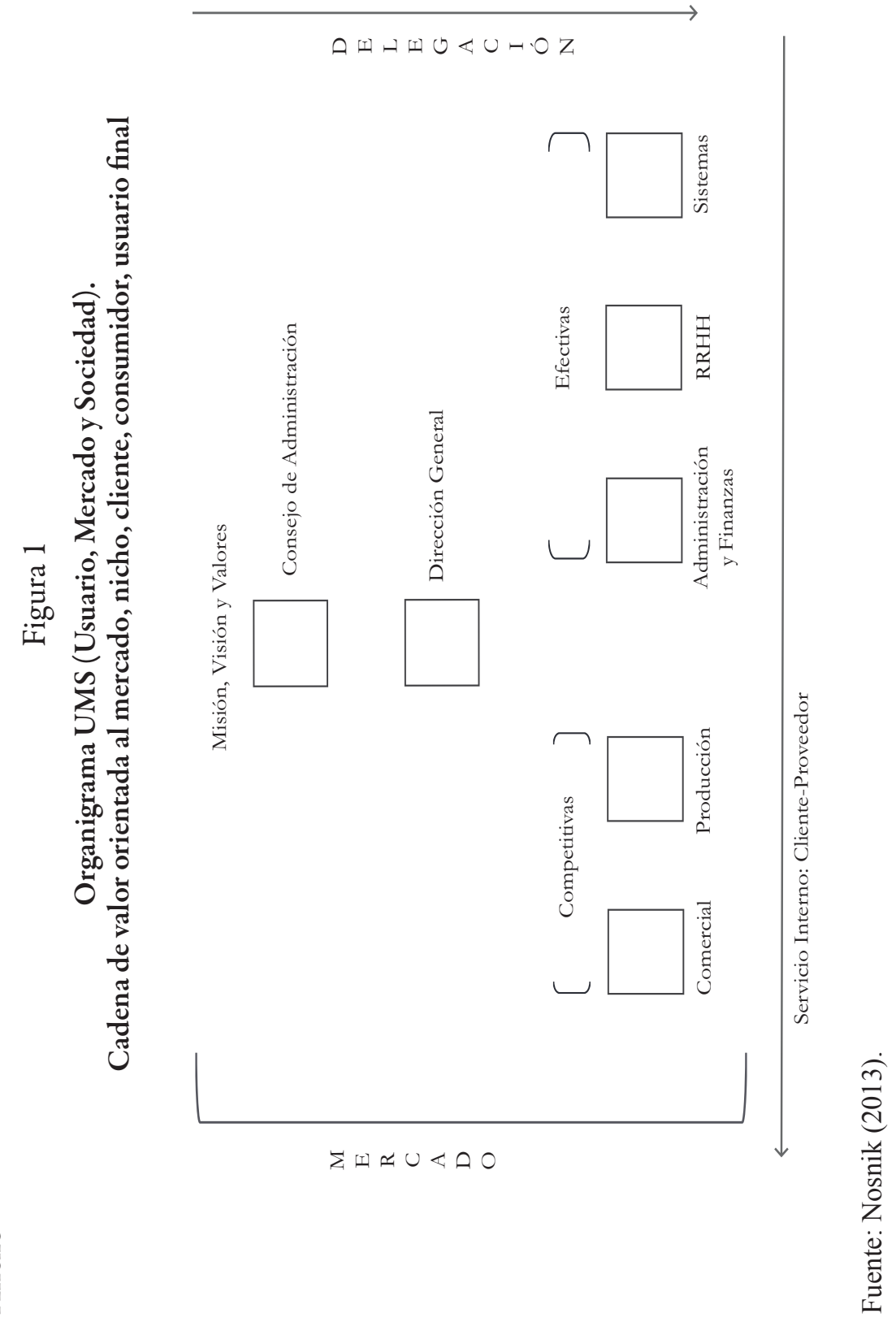




\section{Figura 2}

\section{Modelo UMS aplicado a una institución universitaria}

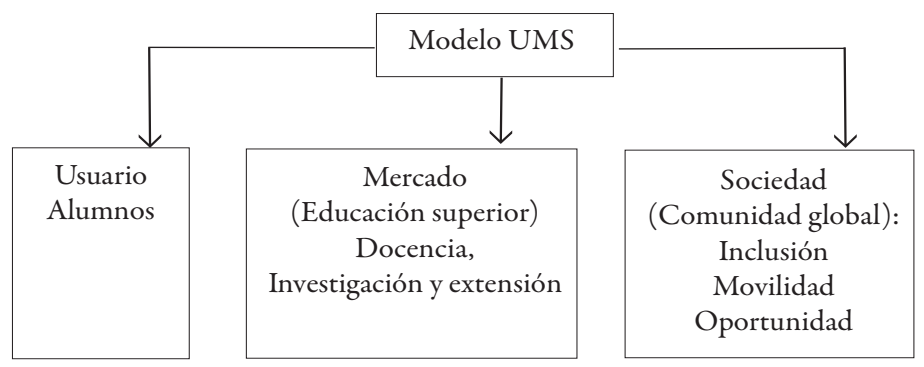

Fuente: Pantoja (2016). 

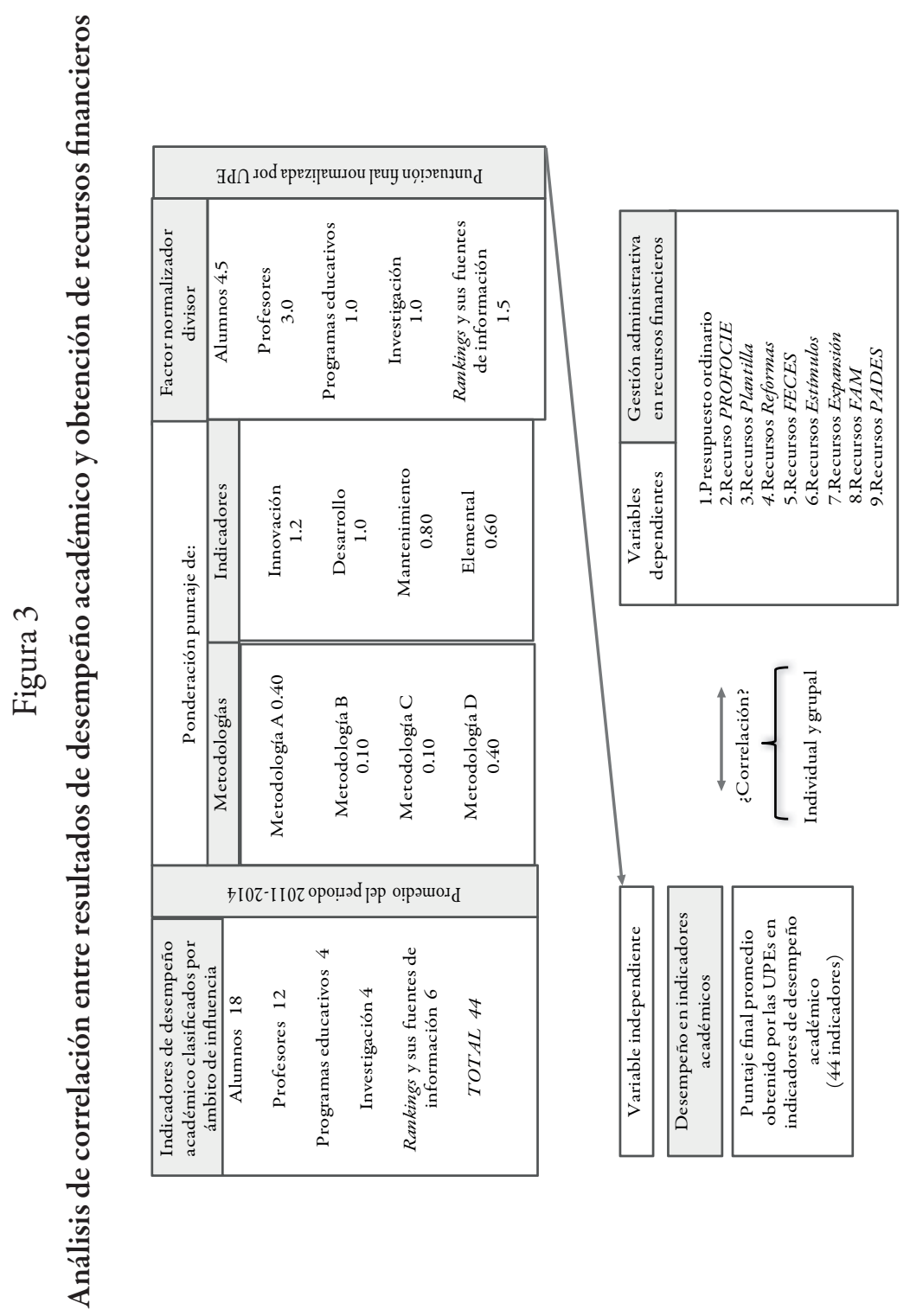

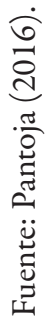


Tabla 1

Coeficientes de correlación $r$ y $r$ s entre la variable independiente puntaje y las variables dependientes

\begin{tabular}{clll}
\hline Núm. & Correlación "puntaje" con: & $\begin{array}{l}\text { Coeficiente } \\
\text { Pearson "r" }\end{array}$ & $\begin{array}{l}\text { Coeficiente } \\
\text { Spearman "rs" }\end{array}$ \\
\hline 1 & Ordinario & 0.117 & 0.075 \\
\hline 2 & PROFOCIE & 0.131 & 0.157 \\
\hline 3 & Plantilla & 0.094 & 0.135 \\
\hline 4 & Reformas & 0.165 & 0.158 \\
\hline 5 & FECES & 0.170 & 0.232 \\
\hline 6 & Estímulos & 0.459 & 0.528 \\
\hline 7 & Expansión & 0.202 & 0.252 \\
\hline 8 & FAM & 0.071 & 0.089 \\
\hline 9 & PADES & 0.197 & 0.184 \\
\hline 10 & Captación total & 0.259 & 0.205 \\
\hline
\end{tabular}

Fuente: Elaboración propia.

Tabla 2

Correlación de la variable independiente y las variables alternas (población por estado y PIBE)

\begin{tabular}{ll}
\hline Coeficiente Pearson "r”: & \\
Indicadores académicos y población por estado & 0.304 \\
\hline Indicadores académicos y PIBE & 0.379 \\
\hline Coeficiente Spearman “rs": & \\
Indicadores académicos y población por estado & 0.272 \\
\hline Indicadores académicos y PIBE & 0.255 \\
\hline
\end{tabular}

Fuente: Elaboración propia. 


\section{Tabla 3}

\section{Análisis de correlación por tercios de grupos de desempeño académico}

\begin{tabular}{|c|c|c|}
\hline Correlación & Coeficiente & Coeficiente \\
\hline "puntaje” con: & Pearson " $r$ " & Spearman "rs" \\
\hline Ordinario & -0.334 & -0.236 \\
\hline PROFOCIE & -0.419 & -0.382 \\
\hline Plantilla & -0.315 & -0.309 \\
\hline Reformas & 0.305 & 0.345 \\
\hline FECES & 0.284 & 0.336 \\
\hline Estímulos & -0.346 & -0.227 \\
\hline Expansión & 0.112 & -0.136 \\
\hline FAM & -0.462 & -0.336 \\
\hline PADES & -0.424 & -0.336 \\
\hline $\begin{array}{l}\text { Captación } \\
\text { total }\end{array}$ & -0.335 & -0.282 \\
\hline
\end{tabular}

Grupo de UPE en el tercio más BAJO en indicadores académicos

\begin{tabular}{lll}
$\begin{array}{ll}\text { Correlación } \\
\text { "puntaje" con: }\end{array}$ & $\begin{array}{l}\text { Coeficien- } \\
\text { te Pearson } \\
\text { "r" }\end{array}$ & $\begin{array}{l}\text { Coeficiente } \\
\text { Spearman } \\
\text { "rs" }\end{array}$ \\
Ordinario & -0.061 & -0.127 \\
PROFOCIE & -0.105 & 0.018 \\
Plantilla & 0.088 & -0.018 \\
Reformas & -0.342 & -0.396 \\
FECES & -0.128 & -0.091 \\
Estímulos & -0.180 & 0.045 \\
Expansión & 0.255 & 0.473 \\
FAM & 0.506 & 0.418 \\
PADES & 0.314 & 0.600 \\
Captación & & \\
total & -0.212 & -0.327 \\
\hline
\end{tabular}

Fuente: Elaboración propia. 
Martín P. Pantoja-Aguilar. Doctor en Liderazgo y Dirección de Instituciones de Educación Superior por la Universidad Anáhuac. MBA por la Southern Oregon University en Estados Unidos. Maestro en Administración por la Shiga University en Japón. Actualmente es profesor investigador del Departamento de Gestión y Dirección de Empresas de la Universidad de Guanajuato. Líneas de investigación: gestión administrativa y financiera empresarial y universitaria, educación internacional y liderazgo en las finanzas y la administración. Publicaciones recientes: Pantoja-Aguilar, Martín P. (2017), "Diferencias y similitudes entre percepción y autopercepción de administradores universitarios y su líder", en Revista Cientifica Pensamiento y Gestión, núm. 43, julio-diciembre, Colombia: Escuela de Negocios de la Universidad del Norte; Pantoja-Aguilar, Martín P. (2017), "El concepto de liderazgo en la historia ante la administración sistémica", en Revista Innovaciones de Negocios, año 14, núm. 27, enero-junio, México: Facultad de Contaduría Pública y Administración de la Universidad Autónoma de Nuevo León; Pantoja-Aguilar, Martín P. (2017), "Comparative study of Leadership competences of female and male Mexican expatriates", en Case Studies in Business and Management, vol. 4, núm. 1, Estados Unidos: Macrothink Institute. 